\title{
ON THE GROWTH OF SOLUTIONS OF CERTAIN LINEAR DIFFERENTIAL EQUATIONS
}

\author{
Simon Hellerstein, Joseph Miles, and John Rossi \\ University of Wisconsin, Department of Mathematics \\ Madison, WI 53706, U.S.A. \\ University of Illinois, Department of Mathematics \\ 1409 W. Green Street, Urbana, IL 61801, U.S.A. \\ Virginia Polytechnic Institute and State University, Department of Mathematics \\ Blacksburg, VA 24061, U.S.A.
}

\begin{abstract}
Suppose $g_{j}, 0 \leq j \leq n-1$, and $h$ are entire functions and that for some $k$, $0 \leq k \leq n-1$, the order of $g_{k}$ does not exceed $\frac{1}{2}$ and does exceed the order of $h$ and the order of all other $g_{j}$. It is shown that then every solution of the differential equation

$$
f^{(n)}+\sum_{j=0}^{n-1} g_{j} f^{(j)}=h
$$

is either a polynomial or an entire function of infinite order. This generalizes a previous result of the authors for second order equations.
\end{abstract}

\section{Introduction}

Let $\varrho(g)$ denote the order of an entire function $g$. In [10], the authors proved the following

Theorem A. Suppose $g_{1}$ and $g_{2}$ are entire functions with $\varrho\left(g_{2}\right)<\varrho\left(g_{1}\right) \leq \frac{1}{2}$. Then any nonconstant (necessarily entire) solution $f$ of

$$
f^{\prime \prime}+g_{1} f^{\prime}+g_{2} f=0
$$

must have infinite order.

It is elementary that the conclusion of Theorem A holds if $\varrho\left(g_{1}\right)<\varrho\left(g_{2}\right)$ for any $\varrho\left(g_{2}\right)$. Easy examples [10] show that the conclusion may fail if $\varrho\left(g_{1}\right)=\varrho\left(g_{2}\right)$ for any value of $\varrho\left(g_{2}\right)$. Elementary examples also show that the conclusion of Theorem A may fail if $\varrho\left(g_{2}\right)<\varrho\left(g_{1}\right)=1$. The possibility of nonconstant solutions of finite order remains open if $\varrho\left(g_{2}\right)<\varrho\left(g_{1}\right) \in\left(\frac{1}{2}, 1\right)$.

The purpose of this paper is to generalize Theorem A to $n^{\text {th }}$ order equations and to remove the restriction that the equation be homogeneous. We prove the following

1991 Mathematics Subject Classification: Primary 34A20; Secondary 30D20, 30D35. 
Theorem 1. Consider the differential equation

$$
f^{(n)}+\sum_{j=0}^{n-1} g_{j} f^{(j)}=h,
$$

where $g_{j}, 0 \leq j \leq n-1$, and $h$ are entire. Suppose there exists $k, 0 \leq k \leq n-1$, such that

$$
\max \left(\varrho(h), \max _{\substack{0 \leq j \leq n-1 \\ j \neq k}} \varrho\left(g_{j}\right)\right):=\varrho^{*}<\varrho\left(g_{k}\right) \leq \frac{1}{2} .
$$

Every solution of (1.1) is either a polynomial or an entire function of infinite order.

It is trivial that every polynomial of degree less than $k$ is a solution of (1.1) for an appropriate choice of $h$.

The conclusion of Theorem 1 also holds if $\varrho^{*}<\mu\left(g_{k}\right) \leq \frac{1}{2}$, where $\mu\left(g_{k}\right)$ is the lower order of $g_{k}$. (See [10, Section V].) For ease of exposition we confine ourselves to the situation considered in the theorem.

Theorem 1 should be compared to recent results of Langley [11, Theorem 2 and Theorem 3] on the exponent of convergence of the zero sets of solutions of homogeneous equations of the form (1.1) in the case that $\varrho\left(g_{k}\right)<\frac{1}{2}$.

Let $m(E)$ denote the Lebesgue measure of a set $E$ of real numbers, and for $E \subset[1, \infty)$, let

$$
m_{\ell}(E)=\int_{E} \frac{d t}{t}
$$

denote the logarithmic measure of $E$. The logarithmic density of $E$ is

$$
\log \operatorname{dens} E=\lim _{r \rightarrow \infty} \frac{m_{\ell}(E \cap[1, r])}{\log r}
$$

provided this limit exists. Typically we shall denote a set of finite logarithmic measure by $E$ and we reserve the notation $E(\alpha), 0 \leq \alpha \leq 1$, for a set specified to have logarithmic density $\alpha$. We do not require that $E$ and $E(\alpha)$ stand for the same set with each occurrence.

We now give an overview of the proof of Theorem 1. We do so not only to provide an outline of the rather intricate proof, but also to point out that a much simplified proof is available in important special cases (for example if $\left.\varrho^{*}<\varrho\left(g_{k}\right)<\frac{1}{2}\right)$.

We suppose that (1.1) has a transcendental solution $f$ of finite order and obtain a contradiction by showing that there are complex numbers $z$ (in fact of arbitrarily large modulus) at which the $j=k$ term of the left side of (1.1) has modulus exceeding the sum of the moduli of all other terms in the equation. 
The proof divides naturally into two cases depending on the behavior of the minimum modulus of $g_{k}\left(r e^{i \theta}\right), 0 \leq \theta \leq 2 \pi$. For an entire $g$, let

$$
\mathscr{L}(r, g)=\min _{|z|=r}|g(z)| .
$$

Crucial to our arguments are the facts contained in the following lemma, based on a special case of a theorem of Drasin and Shea [3, Theorem 8.1] and discussed in detail in [10, Section 2].

Lemma A. Suppose $g$ is entire and $\varrho(g) \leq \frac{1}{2}$. Then either, for every $\varrho<$ $\varrho(g)$, there exists $r_{m} \rightarrow \infty$ such that

$$
\log \mathscr{L}\left(r_{m}, g\right)>r_{m}^{e}
$$

or, for every $\varrho<\varrho(g)$, if

$$
K_{r}(\varrho)=K_{r}=\left\{\theta \in[0,2 \pi): \log \left|g\left(r e^{i \theta}\right)\right|<r^{\varrho}\right\},
$$

there exists a set $E(1) \subset[1, \infty)$ of logarithmic density 1 such that for $r \in E(1)$, $K_{r}$ is an interval (modulo $2 \pi$ ) satisfying

$$
m\left(K_{r}\right) \rightarrow 0, \quad r \in E(1), \quad r \rightarrow \infty
$$

The easier of our two cases (Case I, discussed in Section 3) occurs if (1.3) holds for $g=g_{k}$. In this case we divide (1.1) by $f^{(k)}$ (not identically zero since $f$ is transcendental) and consider the resulting equation

$$
\frac{f^{(n)}}{f^{(k)}}+\sum_{j=0}^{n-1} g_{j} \frac{f^{(j)}}{f^{(k)}}=\frac{h}{f^{(k)}}
$$

at a point $z_{m}=r_{m} e^{i \varphi_{m}}$ of maximum modulus of $f^{(k)}$ on $|z|=r_{m}$ for a sequence $r_{m} \rightarrow \infty$ satisfying (1.3) for some $\varrho \in\left(\varrho^{*}, \varrho\left(g_{k}\right)\right)$.

We note the $j=k$ term of (1.6) is simply $g_{k}$; a lower bound for the modulus of this term at $z_{m}$ (and in fact on all of $|z|=r_{m}$ ) is given by (1.3). The modulus of the terms of (1.6) corresponding to $0 \leq j<k$ as well as the term $h / f^{(k)}$ can be bounded above at $z_{m}$ by (1.2), the fundamental theorem of calculus, and the maximum modulus theorem. The terms of (1.6) corresponding to $k+1 \leq j<n$ as well as the term $f^{(n)} / f^{(k)}$ can be bounded above everywhere on $|z|=r_{m}$ by elementary estimates on the logarithmic derivative (Lemma B, Section 2) provided that $r_{m}$ avoids the small exceptional set $E_{\alpha}$ of Lemma B. The combination of these bounds at $z_{m}$ yields the desired contradiction.

Results of Barry ([1, p. 294] and [2, Theorem 4]) give conditions sufficient to guarantee that $g_{k}$ satisfies (1.3) for a sequence $r_{m} \rightarrow \infty$ disjoint from the 
exceptional set $E_{\alpha}$ of Lemma B. These conditions include $\varrho\left(g_{k}\right)<\frac{1}{2}$ and $\mu\left(g_{k}\right)<$ $\varrho\left(g_{k}\right) \leq \frac{1}{2}$. Thus if $g_{k}$ satisfies either of these conditions, a very short proof of Theorem 1 is available based on Barry's results, the elementary Lemma B, and the brief argument at the beginning of Section 3 ending with (3.8). Full details in Case I (including the fact that if (1.3) holds for some $r_{m} \rightarrow \infty$, then (1.3) holds for a sequence $s_{m} \rightarrow \infty$ where $s_{m}$ is not in the exceptional set $E_{\alpha}$ of Lemma B) appear in Section 3.

Our argument is much more complicated if $g=g_{k}$ satisfies (1.5). The complication in Case II results from the fact that no lower bound for the $j=k$ term of (1.1) is available at points of the form $r e^{i \varphi_{r}}$ for $\varphi_{r} \in K_{r}$. Our approach is to select $\varrho \in\left(\varrho^{*}, \varrho\left(g_{k}\right)\right)$ and to show for a set of $r$-values of logarithmic density 1 contained in the set $E(1)$ of Lemma A and disjoint from the sets $E_{\alpha}$ obtained by applying Lemma B to $f, f^{\prime}, \ldots, f^{(n-1)}$ that there exists $\psi_{r} \in(0,2 \pi)-K_{r}(\varrho)$ such that if $z_{r}=r e^{i \psi_{r}}$, then

$$
\left|f^{(k)}\left(z_{r}\right)\right|>\left|\frac{f^{(k)}\left(z_{r}\right)}{f\left(z_{r}\right)}\right|>\frac{1}{r^{k}}
$$

In Case II we divide (1.1) by $f$ to obtain

$$
\frac{f^{(n)}}{f}+\sum_{j=0}^{n-1} g_{j} \frac{f^{(j)}}{f}=\frac{h}{f}
$$

and find from (1.2), (1.7), and Lemma B that the $j=k$ term of (1.8), evaluated at $z_{r}$, dominates all other terms in the equation, giving the desired contradiction. Note the significance of $\psi_{r} \notin K_{r}$ is that (1.4) provides a lower bound for $\left|g_{k}\left(z_{r}\right)\right|$.

Our proof of the existence of $z_{r}$ satisfying (1.7) is rather complicated. We in fact produce, for $r$ in a set of logarithmic density 1 , a sequence of nonempty sets $P(r, j), 1 \leq j \leq n$, satisfying

$$
\begin{gathered}
(0,2 \pi)-\bar{K}_{r} \supset P(r, 1) \supset P(r, 2) \supset \cdots \supset P(r, n), \\
\left|\frac{f^{(j-1)}\left(r e^{i \theta}\right)}{f^{(j-2)}\left(r e^{i \theta}\right)}\right|>\frac{1}{r}, \quad \theta \in P(r, j), \quad 2 \leq j \leq n,
\end{gathered}
$$

and

$$
\left|f\left(r e^{i \theta}\right)\right|>1, \quad \theta \in P(r, 1) .
$$

It is elementary from (1.9), (1.10), and (1.11) that (1.7) holds at any point $z_{r}=$ $r e^{i \psi_{r}}$ for $\psi_{r} \in P(r, k+1)$. 
We establish the existence of the sets $P(r, j)$ by appealing to a theorem of Radon comparing the total variation, as a point traverses a smooth closed curve, of the argument of the tangent vector to the curve and the total variation of the argument of the point traversing the curve. This comparison is used in conjunction with familiar expressions for these quantities for the curve $f^{(j)}\left(r e^{i \theta}\right), 0 \leq \theta \leq 2 \pi$, in terms of the logarithmic derivatives of $f^{(j)}$ and $f^{(j+1)}$. A second ingredient in the argument producing the sets $P(r, j)$ is an upper bound given in Lemma 4 for

$$
\frac{1}{2 \pi} \int_{K_{r}}\left|\operatorname{Re} \frac{r e^{i \theta} f^{\prime}\left(r e^{i \theta}\right)}{f\left(r e^{i \theta}\right)}\right| d \theta
$$

for entire functions $f$ of finite order. Lemma 4 is an improvement of bounds obtained in [10, Section 4] and may be of independent interest.

The proof of Case II appears in Section 4. It depends on a sizable collection of known or elementary results, all collected in Section 2.

\section{Preliminaries}

We shall find it convenient to use the Ahlfors-Shimizu characteristic $T_{0}(r, f)$ of a meromorphic function, defined by

$$
T_{0}(r, f)=\int_{0}^{r} \frac{A(t, f)}{t} d t
$$

where $A(t, f)$ is the area on the Riemann sphere $\Sigma$ of the image of $|z|<t$ under $f$, with due regard to multiplicity. Since

$$
\left|T_{0}(r, f)-T(r, f)\right|=O(1),
$$

the Nevanlinna and Ahlfors-Shimizu characteristics are interchangeable for many purposes. It is elementary that $A(t, f)$ is a bounded function of $t$ if and only if $f$ is rational. If we denote the length on $\Sigma$ of the curve $\left\{f\left(r e^{i \theta}\right): 0 \leq \theta \leq 2 \pi\right\}$ by $L(r, f)$, it follows [6, p. 144] from the Schwarz inequality for any $\varepsilon>0$ that we have

$$
L(r, f)<A(r, f)^{1 / 2+\varepsilon}, \quad r \notin E,
$$

for some set $E$ with $m_{\ell}(E)<\infty$.

Crucial to our argument in both Case I and Case II is the following upper bound for $\left|f^{\prime}(z) / f(z)\right|$ for entire $f$ of finite order [10, Lemma 4].

Lemma B. Suppose $f$ is a nonconstant entire function of finite order. If $\alpha>0$, there exists a set $E_{\alpha} \subset[1, \infty)$ satisfying

$$
m\left(E_{\alpha} \cap[r / e, e r]\right)<e^{-r^{\alpha}}, \quad r>r_{0}(f),
$$

such that if $|z|=r \notin E_{\alpha}$, then

$$
\left|\frac{f^{\prime}(z)}{f(z)}\right|<e^{r^{2 \alpha}}, \quad r>r_{0}(f) .
$$

In particular $E_{\alpha}$ has finite Lebesgue measure and hence finite logarithmic measure. 
There are many upper estimates for $\left|f^{\prime}(z) / f(z)\right|$ outside of small exceptional sets, certainly including those with much smaller upper bounds than (2.4). (See for example [5], [13], and [14].) We choose the formulation in Lemma B because the very strong information on the intersection of $E_{\alpha}$ with intervals of logarithmic length 2 contained in (2.3) is convenient for our purposes. (See Lemma 6.)

Only Lemma B from this section is needed for the proof of Case I. We now collect several known results which are needed in our proof of Case II. We begin with the following lemma. which follows immediately upon combining Theorem 4 of [9] and Theorem 3 of [8].

Lemma C. Suppose $K>1, \varepsilon>0$, and $\varphi_{j}(t)$ are nondecreasing, nonnegative functions defined on $[1, \infty)$ with

$$
\int_{1}^{r} \frac{\varphi_{1}(t)}{t} d t \leq \int_{1}^{r} \frac{\varphi_{2}(t)}{t} d t+O(1)
$$

for all $r>1$. Then there exists a set $E(1 / K)$ with $\log \operatorname{dens} E(1 / K)=1 / K$ such that

$$
\varphi_{1}(t)<(e+\varepsilon) K \varphi_{2}(t)
$$

for all $t \notin E(1 / K)$.

Our first application of Lemma $\mathrm{C}$ is

Lemma 2. Suppose $f$ is a transcendental entire function of finite order, $\varepsilon>0$, and $j$ is a positive integer. If

$$
h_{j}(z)=z f^{(j)}(z) / f^{(j-1)}(z),
$$

then there exists a set $E_{j}(0) \subset[1, \infty)$ of logarithmic density 0 such that

$$
L\left(r, h_{j}\right)<A(r, f)^{1 / 2+\varepsilon}, \quad r \notin E_{j}(0) .
$$

Proof. By the lemma on the logarithmic derivative, for $r>r_{0}$ we have

$$
\begin{aligned}
T\left(r, h_{j}\right) & =m\left(r, h_{j}\right)+N\left(r, h_{j}\right)<\log r+\varepsilon m(r, f)+N\left(r, 0, f^{(j-1)}\right) \\
& <(1+\varepsilon) m(r, f)+T\left(r, f^{(j-1)}\right)<(2+2 \varepsilon) T(r, f) .
\end{aligned}
$$

From Lemma $C$ and (2.1) we conclude for any $K>1$ that

$$
A\left(r, h_{j}\right)<e K(2+3 \varepsilon) A(r, f), \quad r \notin B_{j}\left(\frac{1}{K}\right)
$$

for some set $B_{j}(1 / K)$ with $\log \operatorname{dens} B_{j}(1 / K)=1 / K$. Since $A(r, f) \rightarrow \infty$, we conclude from (2.2) that

$$
L\left(r, h_{j}\right)<A\left(r, h_{j}\right)^{(1+\varepsilon) / 2}<A(r, f)^{1 / 2+\varepsilon}, \quad r \notin E_{j}\left(\frac{1}{K}\right),
$$

for a set $E_{j}(1 / K)$ with $\log$ dens $E_{j}(1 / K)=1 / K$. Since $K>1$ is arbitrary, the lemma is proved. 
For future use we note that one consequence of Lemma $\mathrm{C}$ and (2.1) is that for nonconstant entire $f$ and $\varepsilon>0$ the inequality

$$
n(r, 0, f)<(e+\varepsilon) K A(r, f)
$$

holds for $r \notin E(1 / K)$ where $\log \operatorname{dens} E(1 / K)=1 / K$.

Our analysis in Case II depends heavily on a comparison between the total variation of the argument of the tangent vector to a smooth closed curve and the total variation of the argument of a point traversing the curve. We need the following lemma, apparently first discovered by Radon [12]. (See also [7, Lemma 4].)

Lemma D. Suppose $\Gamma(\theta), a \leq \theta \leq b$, is a closed analytic curve with $\Gamma(\theta) \neq$ $P_{0}$ and $\Gamma^{\prime}(\theta) \neq 0$. At $P \in \Gamma$, let $\varphi$ and $\psi$ be the angles respectively which $P_{0} P$ and the tangent vector to $\Gamma$ at $P$ make with the positive real axis. Then

$$
\int_{\Gamma}|d \varphi| \leq \int_{\Gamma}|d \psi|
$$

We remark that an examination of the proof shows that the assumption of analyticity may be relaxed to the condition that $\varphi^{\prime}(\theta)=0$ for only a finite number of distinct points $\theta$.

Suppose $f$ is entire. For any $r>0$ with $f^{\prime}\left(r e^{i \theta}\right) f\left(r e^{i \theta}\right) \neq 0$ for $0 \leq \theta \leq 2 \pi$, let $\varphi_{r}(\theta)$ be a continuous argument of $f\left(r e^{i \theta}\right)$ and let $\psi_{r}(\theta)$ be a continuous argument of the tangent vector to the curve $\left\{f\left(r e^{i \theta}\right): 0 \leq \theta \leq 2 \pi\right\}$. Recall that

$$
\varphi_{r}^{\prime}(\theta)=\operatorname{Re} \frac{r e^{i \theta} f^{\prime}\left(r e^{i \theta}\right)}{f\left(r e^{i \theta}\right)}
$$

and

$$
\psi_{r}^{\prime}(\theta)=\operatorname{Re}\left(1+\frac{r e^{i \theta} f^{\prime \prime}\left(r e^{i \theta}\right)}{f^{\prime}\left(r e^{i \theta}\right)}\right) .
$$

Consider $[c, d] \subset[0,2 \pi)$. We join the endpoints of $\left\{f\left(r e^{i \theta}\right): c \leq \theta \leq d\right\}$ with a straight line segment (appropriately smoothed near the endpoints in accordance with the remark following Lemma D) to obtain a closed curve and observe that in so doing we increase the total variation of the argument of the tangent vector by at most $2 \pi$ (in fact by at most $\pi$ at each endpoint). Applying Lemma $\mathrm{D}$, we obtain

$$
\begin{aligned}
\int_{c}^{d}\left|\operatorname{Re} \frac{r e^{i \theta} f^{\prime}\left(r e^{i \theta}\right)}{f\left(r e^{i \theta}\right)}\right| d \theta & \leq \int_{c}^{d}\left|\operatorname{Re}\left(1+\frac{r e^{i \theta} f^{\prime \prime}\left(r e^{i \theta}\right)}{f^{\prime}\left(r e^{i \theta}\right)}\right)\right| d \theta+2 \pi \\
& \leq \int_{c}^{d}\left|\operatorname{Re} \frac{r e^{i \theta} f^{\prime \prime}\left(r e^{i \theta}\right)}{f^{\prime}\left(r e^{i \theta}\right)}\right| d \theta+2 \pi+(d-c) .
\end{aligned}
$$

Inequality (2.7) plays a crucial role in the proof in Case II.

We require the following 
Lemma E. For a nonconstant entire function $f$ and for $m=1,2$, let

$$
\beta_{m}(r)=\frac{1}{2 \pi} \int_{0}^{2 \pi} n\left(r, m e^{i \varphi}, f\right) d \varphi
$$

be the mean covering number of the circle $|z|=m$ under the map $f$ restricted to $|z| \leq r$. There exists a set $E$ with $m_{\ell}(E)<\infty$ such that

$$
\lim _{\substack{r \rightarrow \infty \\ r \notin E}} \frac{\beta_{m}(r)}{A(r, f)}=1
$$

for $m=1,2$.

The proof for $m=1$ appears in [10, Lemma 1], and the $m=2$ case is identical. Lemma $\mathrm{E}$ also follows from Ahlfors's covering surface theory ([6, Theorem 5.2]).

We note that if

$$
B(r, m)=\left\{\theta \in(0,2 \pi): \log \left|f\left(r e^{i \theta}\right)\right|>m\right\},
$$

then from (2.6) and the argument principle it follows that

$$
\beta_{m}(r)=\frac{1}{2 \pi} \int_{B(r, m)} \operatorname{Re}\left(\frac{r e^{i \theta} f^{\prime}\left(r e^{i \theta}\right)}{f\left(r e^{i \theta}\right)}\right) d \theta .
$$

Our final objective in Section 2 is to prove Lemma 4, referred to briefly at the end of Section 1. We begin by establishing the following routine lemma.

Lemma 3. Suppose $n(r)$ is a nondecreasing function of finite order. Suppose $\delta(r) \rightarrow 0$ as $r \rightarrow \infty$. Then there exists $E(0) \subset[1, \infty)$ having logarithmic density 0 such that

$$
n\left(r e^{\delta(r)}\right)-n\left(r e^{-\delta(r)}\right)=o(n(r)), \quad r \notin E(0) .
$$

Proof. Let $\delta>0$ and $\varepsilon>0$. Let

$$
E_{\delta, \varepsilon}=\left\{r>1: n\left(r e^{\delta}\right)-n\left(r e^{-\delta}\right)>\varepsilon n(r)\right\} .
$$

Let $r_{n}=e^{n \delta}, n=0,1,2, \ldots$, and let $I_{n}=\left[r_{n}, r_{n+1}\right)$. Let $J_{N}$ be the number of $n \leq N$ such that $I_{n} \cap E_{\delta, \varepsilon} \neq \phi$. Since

$$
n\left(r e^{\delta}\right)-n\left(r e^{-\delta}\right)>\varepsilon n(r)
$$

implies

$$
\log n\left(r e^{\delta}\right)-\log n\left(r e^{-\delta}\right)>\log (1+\varepsilon)
$$


we conclude for large $N$ that

$$
\left[\frac{J_{N}}{3}\right] \log (1+\varepsilon) \leq \log n\left(r_{N}\right)<(\varrho+1) \log r_{N}=(\varrho+1) N \delta
$$

where $\varrho$ is the order of $n(r)$. Thus $E_{\delta, \varepsilon}$ is contained in a set of logarithmic density at most

$$
\limsup _{N \rightarrow \infty} \frac{J_{N} \delta}{N \delta} \leq \frac{3(\varrho+1) \delta}{\log (1+\varepsilon)}
$$

We may choose $\varepsilon=e^{\sqrt{\delta}}-1$ to conclude that

$$
n\left(r e^{\delta}\right)-n\left(r e^{-\delta}\right) \leq \sqrt{\delta} n(r)
$$

off a set of logarithmic density at most $3(\varrho+1) \sqrt{\delta}$. Since $\delta>0$ is arbitrary, the lemma is established.

Our proof of Lemma 4 requires the following slight strengthening of Lemma 5 of [10]. The strengthening involves obtaining information on a set of large logarithmic density (at least for $K$ large) rather than just on a set of positive logarithmic density. This improvement can easily be achieved by appealing to Theorem 3 of [8] in the very short proof of Lemma 5 in [10].

Lemma F. Let $f$ be an entire function of finite order $\lambda^{\prime}$. Let $\varepsilon>0, K>2$, and $\lambda>\lambda^{\prime}$. Then there exists $E(1-2 / K) \subset[1, \infty)$ of logarithmic density $1-2 / K$ with the property that for $r \in E(1-2 / K)$ there exists $h=h_{r}>0$ such that if $R^{\prime}=r e^{h}$, then

$$
T_{0}\left(R^{\prime}, f\right)<h K(e+\varepsilon) A(r, f)
$$

and

$$
T_{0}\left(R^{\prime}, f\right)<h^{2} K^{2} \lambda(e+\varepsilon) A(r, f)
$$

We are now ready to state

Lemma 4. Let $f$ be entire of finite order and let $K_{r} \subset[0,2 \pi)$ be any measurable set with $m\left(K_{r}\right) \rightarrow 0$ as $r \rightarrow \infty$. Then there exists $E(1) \subset[1, \infty)$ with logarithmic density 1 such that

$$
\int_{K_{r}}\left|\operatorname{Re} \frac{r e^{i \theta} f^{\prime}\left(r e^{i \theta}\right)}{f\left(r e^{i \theta}\right)}\right| d \theta=o(A(r, f)), \quad r \in E(1) .
$$

Obvious modifications of our proof of Lemma 4 show that the conclusion holds for all meromorphic functions of finite order. Since our application is to entire functions, we restrict ourselves to that case for ease of exposition. 
Lemma 4 should be compared to an estimate $[4,(17)]$ for meromorphic functions obtained by Fuchs for the quantity

$$
\int_{J}\left|\frac{r e^{i \theta} f^{\prime}\left(r e^{i \theta}\right)}{f\left(r e^{i \theta}\right)}\right| d \theta
$$

in terms of the measure of the set $J$ and certain functionals related to $f$. While the integral (2.13) is larger than the quantity being estimated in (2.12), the bound for (2.13) obtained in [4] appears to be too large for our purposes.

Since by Lemma $\mathrm{E}$ and (2.9) we have for entire $f$ that

$$
\begin{aligned}
\frac{1}{2 \pi} \int_{0}^{2 \pi}\left(\operatorname{Re} \frac{r e^{i \theta} f^{\prime}\left(r e^{i \theta}\right)}{f\left(r e^{i \theta}\right)}\right)^{+} d \theta & \geq \frac{1}{2 \pi} \int_{B(r, 1)} \operatorname{Re} \frac{r e^{i \theta} f^{\prime}\left(r e^{i \theta}\right)}{f\left(r e^{i \theta}\right)} d \theta \\
& >(1-o(1)) A(r, f), \quad r \notin E, m_{\ell}(E)<\infty,
\end{aligned}
$$

we notice in passing that if $\tilde{K}_{r}=[0,2 \pi)-K_{r}$, Lemma 4 implies for entire $f$ of finite order that

$$
\frac{1}{2 \pi} \int_{\tilde{K}_{r}}\left(\operatorname{Re} \frac{r e^{i \theta} f^{\prime}\left(r e^{i \theta}\right)}{f\left(r e^{i \theta}\right)}\right)^{+} d \theta>(1-o(1)) A(r, f), \quad r \in E(1)-E .
$$

Proof of Lemma 4. We apply the differentiated Poisson-Jensen formula [6, p. 22] to $f$. For $\delta(r)>0$ and $R>r$ to be specified presently, we have with the zeros of $f$ denoted by $a_{n}$ for $|z|=r$ that

$$
\begin{aligned}
\frac{z f^{\prime}(z)}{f(z)}= & \frac{1}{2 \pi} \int_{0}^{2 \pi} \log \left|f\left(\operatorname{Re}^{i \varphi}\right)\right| \frac{2 z \operatorname{Re}^{i \varphi}}{\left(\operatorname{Re}^{i \varphi}-z\right)^{2}} d \varphi \\
& +\sum_{\left|a_{n}\right|<r e^{-\delta(r)}} \frac{z}{z-a_{n}}+\frac{\bar{a}_{n} z}{R^{2}-\bar{a}_{n} z} \\
& +\sum_{r e^{-\delta(r)} \leq\left|a_{n}\right|<r e^{\delta(r)}} \frac{z}{z-a_{n}}+\frac{\bar{a}_{n} z}{R^{2}-\bar{a}_{n} z} \\
& +\sum_{r e^{\delta(r)} \leq\left|a_{n}\right|<R} \frac{z}{z-a_{n}}+\frac{\bar{a}_{n} z}{R^{2}-\bar{a}_{n} z} \\
= & f_{1}(z)+f_{2}(z)+f_{3}(z)+f_{4}(z) .
\end{aligned}
$$

We collect the following observations. Certainly for $|a|<R$ we have

$$
\operatorname{Re}\left(\frac{r e^{i \theta}}{r e^{i \theta}-a}+\frac{\bar{a} r e^{i \theta}}{R^{2}-\bar{a} r e^{i \theta}}\right)=\frac{d}{d \theta} \arg \frac{R\left(r e^{i \theta}-a\right)}{R^{2}-\bar{a} r e^{i \theta}}
$$


and hence

$$
\frac{1}{2 \pi} \int_{0}^{2 \pi}\left|\operatorname{Re}\left(\frac{r e^{i \theta}}{r e^{i \theta}-a}+\frac{\bar{a} r e^{i \theta}}{R^{2}-\bar{a} r e^{i \theta}}\right)\right| d \theta \leq 1 .
$$

If $|a|<r e^{-\delta(r)}$ or if $r e^{\delta(r)}<|a|<R$, then

$$
\left|\frac{r e^{i \theta}}{r e^{i \theta}-a}+\frac{\bar{a} r e^{i \theta}}{R^{2}-\bar{a} r e^{i \theta}}\right| \leq \frac{e^{\delta(r)}+1}{e^{\delta(r)}-1} .
$$

We now specify our choices of $\delta(r)$ and $R>r$ in (2.14). We first choose $\varepsilon>0$ and $K>3$. We next choose $\delta(r) \rightarrow 0$ as $r \rightarrow \infty$ satisfying

$$
\frac{e^{\delta(r)}+1}{e^{\delta(r)}-1} m\left(K_{r}\right) \rightarrow 0
$$

We apply Lemma $\mathrm{F}$ with our given choices of $\varepsilon$ and $K$, and, for $r$ in the set $E(1-2 / K)$ of Lemma $\mathrm{F}$, we choose $R=\sqrt{r R^{\prime}}=r e^{h / 2}$.

We now estimate $(1 / 2 \pi) \int_{K_{r}}\left|\operatorname{Re} f_{j}\left(r e^{i \theta}\right)\right| d \theta$ for $r \in E(1-2 / K)$ and $j=$ $1,2,3,4$. We have from (2.11) that

$$
\begin{aligned}
\frac{1}{2 \pi} \int_{K_{r}} \mid & \operatorname{Re} f_{1}\left(r e^{i \theta}\right) \mid d \theta \leq \frac{(4 T(R, f)+O(1)) e^{h / 2} m\left(K_{r}\right)}{\left(e^{h / 2}-1\right)^{2}} \\
< & \frac{17 T(R, f)}{h^{2}} m\left(K_{r}\right)=o(A(r, f)), \quad r \in E(1-2 / K) .
\end{aligned}
$$

From (2.5), (2.16), and (2.17), we see that there exists a set $E_{1}(1 / K)$ of logarithmic density $1 / K$ such that

$$
\begin{aligned}
\frac{1}{2 \pi} \int_{K_{r}}\left|\operatorname{Re} f_{2}\left(r e^{i \theta}\right)\right| d \theta & \leq \frac{e^{\delta(r)}+1}{e^{\delta(r)}-1} m\left(K_{r}\right) n\left(r e^{-\delta(r)}, 0, f\right) \\
& =o(n(r, 0, f))=o(A(r, f)), \quad r \notin E_{1}(1 / K) .
\end{aligned}
$$

Letting $E(0)$ be the exceptional set of Lemma 3 for $n(r)=n(r, 0, f)$, we see from (2.15) that

$$
\begin{aligned}
\frac{1}{2 \pi} \int_{K_{r}}\left|\operatorname{Re} f_{3}\left(r e^{i \theta}\right)\right| d \theta & \leq n\left(r e^{\delta(r)}, 0, f\right)-n\left(r e^{-\delta(r)}, 0, f\right)=o(n(r, 0, f)) \\
& =o(A(r, f)), \quad r \notin E(0) \cup E_{1}(1 / K) .
\end{aligned}
$$

For $r \in E(1-2 / K)$, we have from (2.10), (2.16), and (2.17) that

$$
\begin{aligned}
\frac{1}{2 \pi} \int_{K_{r}}\left|\operatorname{Re} f_{4}\left(r e^{i \theta}\right)\right| d \theta & \leq n(R, 0, f) \frac{e^{\delta(r)}+1}{e^{\delta(r)}-1} m\left(K_{r}\right) \\
& =o(n(R, 0, f))=o\left(\frac{N\left(R^{\prime}, 0, f\right)}{h}\right) \\
& =o\left(T\left(R^{\prime}, f\right) / h\right)=o(A(r, f)) .
\end{aligned}
$$

Combining (2.18), (2.19), (2.20), and (2.21), we obtain (2.12) for $r \in E(1-2 / K)-$ $\left(E(0) \cup E_{1}(1 / K)\right)$, a set of logarithmic density at least $1-3 / K$. Since $K>3$ is arbitrary, Lemma 4 is proved. 


\section{Case I}

We consider the case where (1.3) holds with $g=g_{k}$ for every $\varrho<\varrho\left(g_{k}\right)$. For simplicity of notation we denote $\varrho\left(g_{k}\right)$ by $\varrho_{k}$ throughout this section. We suppose (1.1) has a transcendental solution $f$ of finite order and seek a contradiction. We choose $\varepsilon>0$ with $17 \varepsilon<\varrho_{k}-\varrho^{*}$. Setting $\alpha=7 \varepsilon$, we apply Lemma B to each of the functions $f^{(k)}, f^{(k+1)}, \ldots, f^{(n-1)}$ to conclude there exists a set $E$ satisfying

$$
m(E \cap[r / e, e r])<e^{-r^{6 \varepsilon}}, \quad r>R_{0}
$$

and if $|z|=r \notin E$,

$$
\left|\frac{f^{(j)}(z)}{f^{(k)}(z)}\right|<e^{r^{15 \varepsilon}}, \quad k+1 \leq j \leq n, r>R_{0}
$$

We show below (Lemma 6 ) that there exists $s_{m} \rightarrow \infty, s_{m} \notin E$ for which

$$
\log \mathscr{L}\left(s_{m}, g_{k}\right)>s_{m}^{\varrho_{k}-2 \varepsilon}, \quad s_{m} \notin E
$$

We recall that Barry ([1], [2]) has given conditions sufficient to guarantee (3.3). Temporarily granting (3.3), we can complete the proof quite quickly.

For convenience we write $g_{n}(z) \equiv 1$ and rearrange (1.1) to obtain

$$
g_{k}=-\left(\sum_{j=k+1}^{n} g_{j} \frac{f^{(j)}}{f^{(k)}}+\sum_{j=0}^{k-1} g_{j} \frac{f^{(j)}}{f^{(k)}}-\frac{h}{f^{(k)}}\right)
$$

Of course if $k=0$ we omit the second sum on the right. Since $s_{m} \notin E$, for all $|z|=s_{m}$ we have by (3.2) that

$$
\left|\sum_{j=k+1}^{n} g_{j}(z) \frac{f^{(j)}(z)}{f^{(k)}(z)}\right| \leq(n-k) \exp \left(s_{m}^{15 \varepsilon}+s_{m}^{\varrho^{*}+\varepsilon}\right), \quad m>m_{0}
$$

Let $z_{m}=r_{m} e^{i \varphi_{m}}$ be such that

$$
M\left(s_{m}, f^{(k)}\right)=\left|f^{(k)}\left(s_{m} e^{i \varphi_{m}}\right)\right|
$$

where $M(r, f)$ denotes the maximum of $|f(z)|$ on $|z|=r$. We note from the fundamental theorem of calculus and the maximum modulus theorem for $0 \leq j \leq$ $k-1$ that

$$
\left|\frac{f^{(j)}\left(z_{m}\right)}{f^{(k)}\left(z_{m}\right)}\right| \leq(1+o(1)) s_{m}^{k-j}
$$


Hence

$$
\left|\sum_{j=0}^{n-1} g_{j}\left(z_{m}\right) \frac{f^{(j)}\left(z_{m}\right)}{f^{(k)}\left(z_{m}\right)}\right| \leq \exp \left(s_{m}^{e^{*}+\varepsilon}\right), \quad m>m_{0}
$$

Since

$$
\left|\frac{h\left(z_{m}\right)}{f^{(k)}\left(z_{m}\right)}\right| \leq \exp \left(s_{m}^{e^{*}+\varepsilon}\right), \quad m>m_{0},
$$

we conclude from (3.4), (3.5), (3.6), and (3.7) that

$$
\left|g_{k}\left(z_{m}\right)\right| \leq \exp \left(s_{m}^{\varrho_{k}-2 \varepsilon}\right), \quad m>m_{0},
$$

contradicting (3.3). Thus the proof in Case I will be finished once we prove Lemma 6.

It should be mentioned that a detailed analysis of the proof of Theorem 8.1 of [3] shows that if for any $\varrho<\varrho(g) \leq \frac{1}{2}$ condition (1.5) does not hold, then there is a set $B$ of infinite measure such that

$$
\log \mathscr{L}(r, g)>r^{\varrho}
$$

for all $r \in B$. Applying this fact with $g=g_{k}$ and observing that the set $E$ where (3.2) may fail has finite measure, we conclude that in fact (3.9) holds with $g=g_{k}$ and each $\varrho<\varrho\left(g_{k}\right)$ for an unbounded set of $r$-values disjoint from $E$. With this observation, the above argument (ending with (3.8)) may be applied to prove the theorem in Case I.

Because of the intricate nature of the proof of Theorem 8.1 of [3], we prefer to give a self-contained argument (Lemma 6) that (3.9) holds with $g=g_{k}$ for an unbounded set disjoint from $E$. We begin with some elementary facts concerning Möbius transformations.

Lemma 5. Suppose $0<r<s$. Let

$$
T(\alpha)=\frac{s-a}{r-a} .
$$

Set $\alpha=R e^{i \theta}$. We have

$$
\text { If } R \leq \sqrt{r s} \text {, then } \min _{0 \leq \theta \leq 2 \pi}\left|T\left(R e^{i \theta}\right)\right|=\frac{s+R}{r+R}
$$

and

$$
\text { if } R>\sqrt{r s} \text {, then } \min _{0 \leq \theta \leq 2 \pi}\left|T\left(R e^{i \theta}\right)\right|=\left|\frac{s-R}{r-R}\right| \text {. }
$$


Proof. Since $T$ maps $|\alpha|=R$ onto a line or circle intersecting the real axis perpendicularly, it is clear that $\min _{0 \leq \theta \leq 2 \pi}\left|T\left(R e^{i \theta}\right)\right|$ occurs either at $\theta=0$ or $\theta=\pi$. Note that

$$
\begin{gathered}
T(-R)=\frac{s+R}{r+R}>0, \\
T(R)=\frac{s-R}{r-R}, \\
T(R)+T(-R)=2 \frac{r s-R^{2}}{r^{2}-R^{2}},
\end{gathered}
$$

and

$$
T(-R)-T(R)=2 R \frac{r-s}{r^{2}-R^{2}} .
$$

We first prove (i). If $r<R \leq \sqrt{r s}$, then $T(R)<0$ and $T(R)+T(-R) \leq$ 0 . Thus $T(-R) \leq|T(R)|$, establishing (i). If $R<r$, then $T(R)>0$ and $T(-R)-T(R)<0$, again implying $T(-R)<T(R)$. If $R=r$, certainly $T(-R)<$ $T(R)=\infty$.

We next prove (ii). If $\sqrt{r s}<R<s$, then $T(R)<0$ and $T(R)+T(-R)>0$. Thus $|T(R)|<T(-R)$, yielding (ii). If $R>s$, then $T(R)>0$ and $T(-R)-$ $T(R)>0$, again implying $T(R)<T(-R)$ and establishing (ii). If $R=s$, (ii) is trivial.

We now state

Lemma 6. Suppose $g=\prod_{\nu}\left(1-z / \alpha_{\nu}\right)$ is entire of order $\lambda<1$ and $0<\varepsilon<$ $\min (\lambda / 2,1-\lambda)$. Suppose there exists an unbounded set of $r$-values such that

$$
\log \mathscr{L}(r, g)>r^{\lambda-\varepsilon} .
$$

Suppose also that $E \subset[1, \infty)$ satisfies

$$
m(E \cap[r / e, e r])<e^{-r^{6 e}}, \quad r>R_{0} .
$$

Then there are arbitrarily large $s \notin E$ such that

$$
\log \mathscr{L}(s, g)>s^{\lambda-2 \varepsilon} .
$$

As previously indicated, Lemma 6 may be applied with $g=g_{k}, \lambda=\varrho\left(g_{k}\right)$, and $E$ as in (3.1) to produce $s_{m} \notin E, s_{m} \rightarrow \infty$ satisfying (3.3).

Proof of Lemma 6. Suppose $r$ satisfies (3.10) and that $n(t, 0, g)<t^{\lambda+\varepsilon}$, $t>r$. Define $r_{j}$ by

$$
r_{j}=r\left(1+2^{j} \exp \left(-r^{5 \varepsilon}\right)\right), \quad 0 \leq j \leq Q,
$$


where $Q=\left[r^{5 \varepsilon} / \log 2\right]$. Let $A_{j}$ denote the annulus

$$
A_{j}=\left\{z: r_{j-1}<|z| \leq r_{j}\right\}, \quad 1 \leq j \leq Q .
$$

Note that

$$
\begin{aligned}
\left\{z: r\left(1+\exp \left(-r^{5 \varepsilon}\right)\right)\right. & \left.<|z|<\frac{3}{2} r\right\} \subset \bigcup_{j=1}^{Q} A_{j} \\
& \subset\left\{z: r\left(1+\exp \left(-r^{5 \varepsilon}\right)\right)<|z| \leq 2 r\right\} .
\end{aligned}
$$

Let $n_{j}$ be the number of zeros of $g$ in $A_{j}$. We have

$$
\sum_{j=1}^{Q} n_{j} \leq(2 r)^{\lambda+\varepsilon}<r^{\lambda+2 \varepsilon}, \quad r>R_{0} .
$$

Consequently there are at most $r^{4 \varepsilon}$ values of $j$ for which $n_{j}>r^{\lambda-2 \varepsilon}$. Since $Q>r^{5 \varepsilon}$, we conclude there exist $\left[r^{\varepsilon}\right]$ consecutive values of $j$ for which $n_{j}<r^{\lambda-2 \varepsilon}$. From this point on we let $j$ denote the first member of such an interval of integers. Thus

$$
n_{j+p}<r^{\lambda-2 \varepsilon}, \quad 0 \leq p \leq\left[r^{\varepsilon}\right]-1 .
$$

Let

$$
a_{j}=\frac{r_{j}-r_{j-1}}{\left(1+n_{j}+n_{j+1}+n_{j+2}+n_{j+3}\right)} .
$$

Let $D_{\nu}$ be the disk of radius $a_{j}$ centered at the zero $\alpha_{\nu}$ of $g$ in $A_{j} \cup A_{j+1} \cup$ $A_{j+2} \cup A_{j+3}$. Note the sum of the diameters of all the $D_{\nu}$ 's is

$$
2 a_{j}\left(n_{j}+n_{j+1}+n_{j+2}+n_{j+3}\right)<2\left(r_{j}-r_{j-1}\right) .
$$

From (3.11) and the fact that

$$
r_{j+2}-r_{j+1}=4\left(r_{j}-r_{j-1}\right) \geq 2 \exp \left(-r^{5 \varepsilon}\right),
$$

we conclude there exists $s \notin E$ such that the circle $|z|=s$ lies in $A_{j+2}$ and does not intersect any $D_{\nu}$. We note explicitly that

$$
|| \alpha_{\nu}|-s| \geq a_{j}
$$

for all $\alpha_{\nu}$ in $A_{j} \cup A_{j+1} \cup A_{j+2} \cup A_{j+3}$. 
It is sufficient to show $\log |g(s)|>s^{\lambda-2 \varepsilon}$. We write $g=G_{1} G_{2} G_{3} G_{4}$ where

$$
\begin{gathered}
G_{1}(z)=\prod_{\left|\alpha_{\nu}\right| \leq \sqrt{r s}}\left(1-\frac{z}{\alpha_{\nu}}\right), \\
G_{2}(z)=\prod_{\sqrt{r s}<\left|\alpha_{\nu}\right|<r_{j+3}}\left(1-\frac{z}{\alpha_{\nu}}\right), \\
G_{3}(z)=\prod_{r_{j+3} \leq\left|\alpha_{\nu}\right|<r_{Q}}\left(1-\frac{z}{\alpha_{\nu}}\right),
\end{gathered}
$$

and

$$
G_{4}(z)=\prod_{r_{Q} \leq\left|\alpha_{\nu}\right|}\left(1-\frac{z}{\alpha_{\nu}}\right) .
$$

We estimate $\log |g(s)|-\log |g(r)|$. Certainly for any $\alpha_{\nu}$,

$$
\log \left|1-\frac{s}{\alpha_{\nu}}\right|-\log \left|1-\frac{r}{\alpha_{\nu}}\right|=\log \left|\frac{s-\alpha_{\nu}}{r-\alpha_{\nu}}\right| .
$$

Applying Lemma 5 we conclude

$$
\log \left|G_{1}(s)\right|-\log \left|G_{1}(r)\right| \geq \sum_{\left|\alpha_{\nu}\right| \leq \sqrt{r s}} \log \frac{s+\left|\alpha_{\nu}\right|}{r+\left|\alpha_{\nu}\right|} \geq 0 .
$$

Now suppose $G_{2}\left(\alpha_{\nu}\right)=0$. Thus

$$
\left|\alpha_{\nu}\right|>\sqrt{r s}>\sqrt{r r_{j+1}}=r\left(1+2^{j+1} \exp \left(-r^{5 \varepsilon}\right)\right)^{1 / 2}>r_{j-1}
$$

and hence $\alpha_{\nu} \in A_{j} \cup A_{j+1} \cup A_{j+2} \cup A_{j+3}$. Since

$$
\left|a_{\nu}\right|-r \leq r_{j+3}-r=2^{j+3} r \exp \left(-r^{5 \varepsilon}\right)=16\left(r_{j}-r_{j-1}\right),
$$

we conclude from (3.14), (3.15), and Lemma 5(ii) that

$$
\begin{aligned}
\log \left|1-\frac{s}{\alpha_{\nu}}\right|-\log \left|1-\frac{r}{\alpha_{\nu}}\right| & \geq \log \left|\frac{s-\left|\alpha_{\nu}\right|}{r-\left|\alpha_{\nu}\right|}\right| \geq \log \frac{a_{j}}{16\left(r_{j}-r_{j-1}\right)} \\
& =\log \frac{1}{16\left(1+n_{j}+n_{j+1}+n_{j+2}+n_{j+3}\right)} .
\end{aligned}
$$

Thus from (3.13) we have

$$
\begin{aligned}
\log \left|G_{2}(s)\right|-\log \left|G_{2}(r)\right| \geq & \left(n_{j}+n_{j+1}+n_{j+2}+n_{j+3}\right) \times \\
& \times \log \frac{1}{16\left(1+n_{j}+n_{j+1}+n_{j+2}+n_{j+3}\right)} \\
\geq & -4 r^{\lambda-2 \varepsilon} \log (128+(\lambda-2 \varepsilon) \log r) .
\end{aligned}
$$


Now suppose $G_{3}\left(a_{\nu}\right)=0$. Noting

$$
\frac{s-r}{\left|\alpha_{\nu}\right|-r} \leq \frac{r_{j+2}-r}{r_{j+3}-r}=\frac{1}{2}
$$

we apply Lemma 5(ii) to conclude

$$
\begin{aligned}
\log \left|1-\frac{s}{\alpha_{\nu}}\right|-\log \left|1-\frac{r}{\alpha_{\nu}}\right| & \geq \log \frac{\left|\alpha_{\nu}\right|-s}{\left|\alpha_{\nu}\right|-r} \\
& =\log \left(1-\frac{s-r}{\left|a_{\nu}\right|-r}\right)>-2 \frac{s-r}{\left|a_{\nu}\right|-r} .
\end{aligned}
$$

Thus

$$
\begin{aligned}
\log \left|G_{3}(s)\right|- & \log \left|G_{3}(r)\right| \geq-2 \sum_{p=4}^{Q-j}\left\{\sum_{\alpha_{\nu} \in A_{p+j}} \frac{s-r}{\left|\alpha_{\nu}\right|-r}\right\} \\
& \geq-2 \sum_{p=4}^{Q-j} n_{p+j} \frac{r_{j+2}-r}{r_{p+j-1}-r} \\
& =-2 \sum_{p=4}^{Q-j} n_{p+j} \frac{2^{j+2} r \exp \left(-r^{5 \varepsilon}\right)}{2^{j+p-1} r \exp \left(-r^{5 \varepsilon}\right)} \\
& =-2 \sum_{p=4}^{Q-j} n_{p+j} 2^{3-p} \\
& =-2 \sum_{p=4}^{\left[r^{\varepsilon}\right]-1} n_{p+j} 2^{3-p}-2 \sum_{p=\left[r^{\varepsilon}\right]}^{Q-j} n_{p+j} 2^{3-p} \\
& >-2 r^{\lambda-2 \varepsilon}-32 r^{\lambda+2 \varepsilon_{2}-\left[r^{\varepsilon}\right]}>-3 r^{\lambda-2 \varepsilon}, \quad r>R_{0},
\end{aligned}
$$

where in the last step we have used (3.13) and the fact that $n_{p+j}<r^{\lambda+2 \varepsilon}$ for all $p \leq Q-j$.

To analyze the contribution from $G_{4}$, we first define annuli

$$
B_{q}=\left\{z: 2^{q-1} r_{Q}<|z| \leq 2^{q} r_{Q}\right\}
$$

for $q=1,2,3, \ldots$ If $m_{q}$ denotes the number of zeros of $G_{4}$ in $B_{q}$, then certainly

$$
m_{q}<\left(2^{q} r_{Q}\right)^{\lambda+\varepsilon}<\left(2^{q+1} r\right)^{\lambda+\varepsilon} .
$$

Since by our choice of $j$ we have $j+\left[r^{\varepsilon}\right]-1 \leq Q$, we conclude

$$
r_{j+\left[r^{\varepsilon}\right]-1}=r\left(1+2^{j+\left[r^{\varepsilon}\right]-1} \exp \left(-r^{5 \varepsilon}\right)\right) \leq 2 r,
$$


and hence

$$
2^{j} \exp \left(-r^{5 \varepsilon}\right) \leq 2^{1-\left[r^{\varepsilon}\right]}
$$

If $G_{4}\left(\alpha_{\nu}\right)=0$, we have as in (3.18) that

$$
\log \left|1-\frac{s}{\alpha_{\nu}}\right|-\log \left|1-\frac{r}{\alpha_{\nu}}\right|>-2 \frac{s-r}{\left|\alpha_{\nu}\right|-r} .
$$

Suppose $\alpha_{\nu} \in B_{q}$. From (3.21) we have

$$
\log \left|1-\frac{s}{\alpha_{\nu}}\right|-\log \left|1-\frac{r}{\alpha_{\nu}}\right|>-2 \frac{2^{j+2} r \exp \left(-r^{5 \varepsilon}\right)}{2^{q-2} r}>-2^{6-q-\left[r^{\varepsilon}\right]} .
$$

From (3.20), (3.22), and the fact that $\lambda+\varepsilon<1$, we conclude

$$
\begin{aligned}
\log \left|G_{4}(s)\right|-\log \left|G_{4}(r)\right| & \geq-\sum_{q=1}^{\infty} m_{q} 2^{6-q-\left[r^{c}\right]} \\
& >-128 r^{\lambda+\varepsilon} 2^{-\left[r^{\varepsilon}\right]} \sum_{q=1}^{\infty} 2^{q(\lambda+\varepsilon-1)}=o(1) .
\end{aligned}
$$

Combining (3.10), (3.16), (3.17), (3.19), and (3.23), we have

$$
\begin{aligned}
\log |g(s)| & >\log |g(r)|-4 r^{\lambda-2 \varepsilon} \log (128+(\lambda-2 \varepsilon) \log r)-3 r^{\lambda-2 \varepsilon}-O(1) \\
& >s^{\lambda-2 \varepsilon}, \quad s>s_{0} .
\end{aligned}
$$

Thus $s$ satisfies (3.12) and the lemma is proved.

\section{Case II}

We suppose (1.1) has a transcendental solution $f$ of finite order, $\varepsilon>0$ satisfies $5 \varepsilon<\varrho\left(g_{k}\right)-\varrho^{*}$, and that the set

$$
K_{r}\left(\varrho\left(g_{k}\right)-\varepsilon\right)=K_{r}=\left\{\theta \in[0,2 \pi): \log \left|g_{k}\left(r e^{i \theta}\right)\right|<r^{\varrho\left(g_{k}\right)-\varepsilon}\right\}
$$

is an interval (modulo $2 \pi$ ) satisfying $m\left(K_{r}\right) \rightarrow 0$ as $r \rightarrow \infty, r \in E(1)$ for some set $E(1)$ of logarithmic density 1 . We seek a contradiction. By applying Lemma B with $\alpha=\varepsilon$ to each of the functions $f^{(m)}, 0 \leq m \leq n-1$, we may also presume that

$$
\left|\frac{f^{(j)}(z)}{f(z)}\right|<e^{r^{3 e}}, \quad 1 \leq j \leq n
$$


for $|z|=r \in E(1)$.

Recalling definition (2.8), we let

$$
Y(r, m)=B(r, m)-\bar{K}_{r}
$$

Appealing to (2.9), Lemma E, and Lemma 4, we may also presume $E(1)$ to be such that for $m=1,2$ we have

$$
\begin{aligned}
\frac{1}{2 \pi} \int_{Y(r, m)}\left(\operatorname{Re} \frac{r e^{i \theta} f^{\prime}\left(r e^{i \theta}\right)}{f\left(r e^{i \theta}\right)}\right)^{+} d \theta & \geq \frac{1}{2 \pi} \int_{B(r, m)} \operatorname{Re} \frac{r e^{i \theta} f^{\prime}\left(r e^{i \theta}\right)}{f\left(r e^{i \theta}\right)} d \theta-o(A(r, f)) \\
& \geq \frac{1}{2 \pi} \int_{0}^{2 \pi} n\left(r, m e^{i \varphi}, f\right) d \varphi-o(A(r, f)) \\
& \geq(1-o(1)) A(r, f), \quad r \in E(1) .
\end{aligned}
$$

We write

$$
B(r, 1)-\bar{K}_{r}=\bigcup_{j=1}^{\alpha_{r}} I_{j},
$$

where the $I_{j}$ are disjoint open intervals. Let

$$
D(r)=\left\{j: 1 \leq j \leq \alpha_{r} \text { and } I_{j} \cap B(r, 2) \neq \phi\right\},
$$

and let

$$
P(r, 1)=\bigcup_{j \in D(r)} I_{j} .
$$

We note that $P(r, 1)$ satisfies (1.9) and (1.11). Since

$$
P(r, 1) \supset B(r, 2)-\bar{K}_{r}=Y(r, 2),
$$

we conclude from (4.2) that

$$
\begin{aligned}
\frac{1}{2 \pi} \int_{P(r, 1)}\left(\operatorname{Re} \frac{r e^{i \theta} f^{\prime}\left(r e^{i \theta}\right)}{f\left(r e^{i \theta}\right)}\right)^{+} d \theta & \geq \frac{1}{2 \pi} \int_{Y(r, 2)}\left(\operatorname{Re} \frac{r e^{i \theta} f^{\prime}\left(r e^{i \theta}\right)}{f\left(r e^{i \theta}\right)}\right)^{+} d \theta \\
& \geq(1-o(1)) A(r, f), \quad r \in E(1) .
\end{aligned}
$$

Either $\alpha_{r}=1$ or else, for each $j \in D(r), I_{j}$ has an endpoint in $\delta B(r, 1)$ and hence the total length on $\Sigma$ of the curve $\left\{f\left(r e^{i \theta}\right): \theta \in I_{j}\right\}$ is at least the spherical distance from 1 to 2 , namely $1 / \sqrt{10}$. By $(2.2)$, the number of elements of $D(r)$ is at most $\sqrt{10} A(r, f)^{1 / 2+\varepsilon}$ for $r \in E(1), r>R_{0}$. We denote those $I_{j}$ with $j \in D(r)$ by $U_{j}, 1 \leq j \leq \alpha_{r}^{\prime}$, where

$$
\alpha_{r}^{\prime} \leq \sqrt{10} A(r, f)^{1 / 2+\varepsilon}, \quad r \in E(1), r>R_{0}
$$


For $m=1,2$ we now define

$$
C(r, m)=\left\{\theta \in(0,2 \pi):\left|\frac{r e^{i \theta} f^{\prime}\left(r e^{i \theta}\right)}{f\left(r e^{i \theta}\right)}\right|>m\right\}
$$

and write

$$
C(r, 1) \cap P(r, 1)=\bigcup_{j=1}^{\beta_{r}} H_{j},
$$

where the $H_{j}$ are disjoint open intervals. Let

$$
Q(r)=\left\{j: 1 \leq j \leq \beta_{r} \text { and } H_{j} \cap C(r, 2) \neq \emptyset\right\} .
$$

For $j \in Q(r)$, either $H_{j}$ is some $U_{p}, 1 \leq p \leq \alpha_{r}^{\prime}$, or, since one endpoint of $H_{j}$ lies in $\delta C(r, 1)$, the curve $\left\{r e^{i \theta} f^{\prime}\left(r e^{i \theta}\right) / f\left(r e^{i \theta}\right): \theta \in H_{j}\right\}$ has length on $\Sigma$ at least $1 / \sqrt{10}$. We denote those $H_{j}$ with $j \in Q(r)$ by $V_{j}, 1 \leq j \leq \beta_{r}^{\prime}$, and from (4.4) and Lemma 2 (applied to $z f^{\prime}(z) / f(z)$ ) note that

$$
\beta_{r}^{\prime} \leq \alpha_{r}^{\prime}+\sqrt{10} A(r, f)^{1 / 2+\varepsilon} \leq 2 \sqrt{10} A(r, f)^{1 / 2+\varepsilon}, \quad r \in E(1), r>R_{0} .
$$

We write

$$
P(r, 2)=\bigcup_{j=1}^{\beta_{r}^{\prime}} V_{j}
$$

and let $\tilde{C}(r, 2)=(0,2 \pi)-C(r, 2)$. Clearly we have

$$
P(r, 1)-\tilde{C}(r, 2) \subset P(r, 2) \subset P(r, 1) \cap C(r, 1) .
$$

Certainly $P(r, 2)$ satisfies (1.9) and (1.10). Since

$$
\frac{1}{2 \pi} \int_{\tilde{C}(r, 2)}\left|\frac{r e^{i \theta} f^{\prime}\left(r e^{i \theta}\right)}{f\left(r e^{i \theta}\right)}\right| d \theta \leq 2
$$

we deduce from (4.3) that

$$
\frac{1}{2 \pi} \int_{P(r, 2)}\left(\operatorname{Re} \frac{r e^{i \theta} f^{\prime}\left(r e^{i \theta}\right)}{f\left(r e^{i \theta}\right)}\right)^{+} d \theta \geq(1-o(1)) A(r, f), \quad r \in E(1) .
$$

For $1 \leq j \leq \beta_{r}^{\prime}$, we join the endpoints of $\left\{f\left(r e^{i \theta}\right): \theta \in V_{j}\right\}$ with a line segment (smoothed at the endpoints), apply (2.7), and sum from $j=1$ to $j=\beta_{r}^{\prime}$ to obtain

$$
\int_{P(r, 2)}\left|\operatorname{Re} \frac{r e^{i \theta} f^{\prime}\left(r e^{i \theta}\right)}{f\left(r e^{i \theta}\right)}\right| d \theta \leq \int_{P(r, 2)}\left|\operatorname{Re} \frac{r e^{i \theta} f^{\prime \prime}\left(r e^{i \theta}\right)}{f^{\prime}\left(r e^{i \theta}\right)}\right| d \theta+2 \pi+2 \pi \beta_{r}^{\prime} .
$$


From (4.6), (4.8), and (4.9) we deduce

$$
\frac{1}{2 \pi} \int_{P(r, 2)}\left|\operatorname{Re} \frac{r e^{i \theta} f^{\prime \prime}\left(r e^{i \theta}\right)}{f^{\prime}\left(r e^{i \theta}\right)}\right| d \theta \geq(1-o(1)) A(r, f), \quad r \in E(1) .
$$

We note for emphasis that our analysis requires control $\left(\beta_{r}^{\prime}=o(A(r, f))\right)$ of the number of disjoint open intervals comprising $P(r, 2)$ in order to deduce (4.10) from (4.9). We also note that the fact that $f$ is transcendental (and hence $A(r, f) \rightarrow \infty$ ) is used in obtaining (4.10).

Crucial to our argument is that $P(r, 2)$ satisfies $(1.10),(4.6),(4.7)$, and (4.10). We now repeat the above reasoning to produce $P(r, 3) \subset P(r, 2)$ satisfying appropriate analogues of these inequalities. We begin at stage (4.5) by defining

$$
C^{*}(r, m)=\left\{\theta \in(0,2 \pi):\left|\frac{r e^{i \theta} f^{\prime \prime}\left(r e^{i \theta}\right)}{f^{\prime}\left(r e^{i \theta}\right)}\right|>m\right\}
$$

for $m=1,2$. We proceed from (4.5) as before, now replacing $f$ by $f^{\prime}, P(r, 1)$ by $P(r, 2), \alpha_{r}^{\prime}$ by $\beta_{r}^{\prime},(4.4)$ by $(4.6)$, and (4.3) by (4.10). Our argument requires an application of Lemma 2 to the function $z f^{\prime \prime}(z) / f^{\prime}(z)$, and at an appropriate stage we join the endpoints of intervals of the form $\left\{f^{\prime}\left(r e^{i \theta}\right): \theta \in V_{j}^{*}\right\}$ with line segments. We ultimately obtain a set $P(r, 3) \subset P(r, 2) \cap C^{*}(r, 1)$ satisfying analogues of (1.10), (4.6), (4.7), and (4.10). Explicitly, $P(r, 3)$ is expressible as a disjoint union of at most $3 \sqrt{10} A(r, f)^{1 / 2+\varepsilon}$ open intervals and satisfies

$$
\frac{1}{2 \pi} \int_{P(r, 3)}\left|\operatorname{Re} \frac{r e^{i \theta} f^{\prime \prime \prime}\left(r e^{i \theta}\right)}{f^{\prime \prime}\left(r e^{i \theta}\right)}\right| d \theta \geq(1-o(1)) A(r, f), \quad r \in E(1)
$$

and

$$
\left|\frac{r e^{i \theta} f^{\prime \prime}\left(r e^{i \theta}\right)}{f^{\prime}\left(r e^{i \theta}\right)}\right|>1, \quad \theta \in P(r, 3) .
$$

Continuing the iteration, we obtain for $r \in E(1)$ sets $P(r, j), 1 \leq j \leq$ $n$, expressible as a disjoint union of at most $j \sqrt{10} A(r, f)^{1 / 2+\varepsilon}$ open intervals, satisfying

$$
\begin{array}{r}
P(r, n) \subset P(r, n-1) \subset \cdots \subset P(r, 2) \subset P(r, 1) \subset B(r, 1)-\bar{K}_{r}, \\
\frac{1}{2 \pi} \int_{P(r, j)}\left|\operatorname{Re} \frac{r e^{i \theta} f^{(j)}\left(r e^{i \theta}\right)}{f^{(j-1)}\left(r e^{i \theta}\right)}\right| d \theta \geq(1-o(1)) A(r, f), \quad 1 \leq j \leq n
\end{array}
$$

and

$$
\left|\frac{r e^{i \theta} f^{(j-1)}\left(r e^{i \theta}\right)}{f^{(j-2)}\left(r e^{i \theta}\right)}\right|>1, \quad \theta \in P(r, j), 2 \leq j \leq n
$$


Note that the sets $P(r, j)$ satisfy (1.9), (1.10), and (1.11).

We rearrange (1.1) to obtain

$$
\frac{f^{(k)}}{f}=\frac{\frac{f^{(n)}}{f}+\sum_{j \neq k} g_{j} \frac{f^{(j)}}{f}}{g_{k}\left(\frac{h}{g_{k} f^{(k)}}-1\right)}
$$

For $\theta_{k} \in P(r, k+1)$ it follows from (4.11) and (4.12) that

$$
\left|\frac{f^{(k)}\left(r e^{i \theta_{k}}\right)}{f\left(r e^{i \theta_{k}}\right)}\right|=\frac{1}{r^{k}}\left|\prod_{j=2}^{k+1} r e^{i \theta_{k}} \frac{f^{(j-1)}\left(r e^{i \theta_{k}}\right)}{f^{(j-2)}\left(r e^{i \theta_{k}}\right)}\right|>\frac{1}{r^{k}}, \quad r \in E(1) .
$$

Since $\theta_{k} \in B(r, 1)$, we also have

$$
\left|f^{(k)}\left(r e^{i \theta_{k}}\right)\right|>r^{-k}, \quad r \in E(1) .
$$

Since $\theta_{k} \notin K_{r}$, for $z=r e^{i \theta_{k}}$ we conclude by our choice of $\varepsilon>0$ that for large $r \in E(1)$

$$
\left|g_{k}(z)\left(\frac{h(z)}{g_{k}(z) f^{(k)}(z)}-1\right)\right|>\frac{1}{2}\left|g_{k}(z)\right|>\frac{1}{2} \exp \left(r^{\varrho\left(g_{k}\right)-\varepsilon}\right) .
$$

For $|z|=r$, we have from (4.1) for large $r \in E(1)$ that

$$
\left|\frac{f^{(n)}(z)}{f(z)}+\sum_{j \neq k} g_{j}(z) \frac{f^{(j)}(z)}{f(z)}\right|<\exp \left(r^{\varrho^{*}+\varepsilon}+r^{4 \varepsilon}\right) .
$$

The combination of (4.13), (4.14), (4.15), and (4.16) with $z=r e^{i \theta_{k}}, \theta_{k} \in$ $P(r, k+1)$, implies for all large $r \in E(1)$ that

$$
r^{-k}<2 \exp \left(r^{\varrho^{*}+\varepsilon}+r^{4 \varepsilon}-r^{\varrho\left(g_{k}\right)-\varepsilon}\right)
$$

which is the desired contradiction. 


\section{References}

[1] Barry, P.: On a theorem of Besicovitch. - Quart. J. Math. Oxford (2) 14, 1963, 293-302.

[2] BARRY, P.: Some theorems related to the $\cos \pi \varrho$ theorem. - Proc. London Math. Soc. (3) $21,1970,334-360$.

[3] Drasin, D., and D. Shea: Pólya peaks and the oscillation of positive functions. - Proc. Amer. Math. Soc. 34, 1972, 403-411.

[4] Fuchs, W.: Proof of a conjecture of G. Pólya concerning gap series. - Ill. J. Math. 7, 1963, 661-667.

[5. Gundersen, G.: Estimates for the logarithmic derivative of a meromorphic function plus similar estimates. - J. London Math. Soc. (2) 37, 1988, 88-104.

[6] Hayman, W.: Meromorphic functions. - Clarendon Press, Oxford, 1964.

[7] HaYMAN, W., and J. Miles: On the growth of a meromorphic function and its derivatives. - Complex Variables Theory Appl. 12, 1989, 245-260.

[8] Hayman, W., and J. Rossi: Characteristic, maximum modulus, and value distribution. - Trans. Amer. Math. Soc. 284, 1984, 651-664.

[9] Hayman, W., and F. Stewart: Real inequalities with applications to function theory. Proc. Cambridge Philos. Soc. 50, 1954, 250-260.

[10] Hellerstein, S., J. Miles, and J. Rossi: On the growth of solutions of $f^{\prime \prime}+g f^{\prime}+h f=$ 0. - Trans. Amer. Math. Soc. 324, 1991, 693-706.

[11] LANGLEY, J.: Some oscillation theorems for higher order linear differential equations with entire coefficients of small growth. - Results in Math. 20, 1991, 517-529.

[12] Radon, J.: Über die Randwertaufgaben beim logarithmischen Potential. - Sitzungsber. Akad. Wiss. Wien 128, 1919, 1123-1167.

[13] Strelitz, S.: Upper bounds for the logarithmic derivative of a meromorphic function and the existence of maximal growth solutions of linear differential equations. - Preprint.

[14] Valiron, G.: Lectures on the general theory of integral functions. - Chelsea, New York, 1949.

Received 2 December 1991 